\title{
Aproximación teórica al Síndrome de Munchausen por Poderes.
}

Theoretical approach to Munchausen syndrome by proxy.

Marta Cedenilla Vecina (1)

Ana Jiménez-Perianes (2)

(1) Universidad CEU San Pablo, Madrid, España.

(2) Universidad CEU San Pablo, Madrid, España.

Email de correspondencia: m.cedenilla.vecina@gmail.com

\section{Resumen}

El Sindrome de Munchausen por Poderes (SMP) es una forma de maltrato infantil poco frecuente que causa una elevada morbimortalidad, teniendo un dificil diagnóstico y manejo de la situación posterior. El niño es víctima de una enfermedad inducida o simulada por uno de los padres, generalmente la madre, o cuidadores, sometiéndole a diversos estudios médicos, tratamientos innecesarios, hospitalizaciones y procedimientos diagnósticos invasivos y perjudiciales para el niño. Se realiza una revisión de los distintos estudios acerca del SMP en las últimas décadas del s.XX y las primeras del s. XXI.

\section{Palabras Clave}

Sindrome de Munchausen por poderes, maltrato infantil, Trastorno Facticio aplicado a otro, figura perpetradora, simulación, falsificación, Trastorno Facticio por poderes.

\begin{abstract}
Munchausen syndrome by proxy (MSBP) is an infrequent form of child abuse that causes high rate of morbidity and mortality, having a difficult diagnosis and manage the subsequent situation. The child is the victim of a disease induced or simulated by one of the parents, generally the mother, or caregivers, subjecting him to various medical studies, unnecessary treatments, hospitalizations and invasive diagnostic procedures that are harmful to the child. $A$ review of the different studies on MSBP in the last decades of the 20th century and the first decades of the 21st century.
\end{abstract}

\section{Keywords}

Munchausen Syndrome by proxy, child abuse, Factitious Disorder applied to another, perpetrator figure, simulation, falsification, Factitious Disorder by proxy. 


\section{INTRODUCCIÓN}

El Síndrome de Munchausen (SM) se caracteriza por pacientes que falsifican sus propios síntomas (Maida, Molina \& Carrasco, 1999). Meadow (1977) acuñó el término Síndrome de Munchausen por Poderes (SMP), y según de la Cerda et al. (2006), Garrote et al. (2008) y Goñi et al. (2008) explican que se define como aquella persona que inventa ciertos síntomas en otro. Verity, Winckworth, Burman, Stevens, White (1977) lo denominan Sindrome de Polle en el que la madre, que sufre este síndrome, lo induce a sus hijos, a través de distintas formas pudiendo darse de manera física o psicológica.

Asher (1951) en su encuadre para establecer el concepto del síndrome, hace referencia a dos actores: por una parte, hace referencia al paciente con la intención de mentir a otra persona $y$, por otra parte, el médico con sus aportaciones clínicas. Al introducir Meadow (1977) el término por poder incluye a un tercer actor, quién es el responsable de la provocación e invención de síntomas en el niño, generalmente el cuidador principal, con el objetivo de satisfacer sus necesidades psicológicas.

Dentro de la clasificación del manual DSM-IV-TR (APA, 2002) el SMP no aparece como tal, por lo que se usa el término "Desorden Facticio por Poderes" como definición (de la Cerda et al. 2010; Goñi et al., 2008) y se encuentra dentro de la clasificación de Trastornos Facticios no especificado (APA, 2002; Rodríguez et al., 2003).

Por lo tanto, el SMP es un trastorno que abarca las conceptualizaciones anteriormente descritas y se compone de dos elementos básicos: un diagnóstico en el niño y otro en el cuidador. Solo cuando la motivación es la satisfacción de las necesidades psicológicas de la madre, se emplearía el término de SMP, implicando una situación de malos tratos que puede abarcar desde la falsificación de síntomas hasta la producción intencionada del daño físico a través de distintos medios (APA, 2002; Cujiño et al., 2010; de la Cerda et al., 2006; Shaw et al., 2008).

En el presente artículo se ofrece una revisión clínica de los diferentes estudios sobre el SMP atendiendo a las distintas variables de relevancia clínica y forense que componen este trastorno. Así mismo, se presenta una revisión descriptiva de los principales conceptos en torno al SMP.
Se realizó una búsqueda en bases de datos tales como Medline, Psicodoc, Psycinfo, Psyke, Dialnet, Scopus, Scielo, Google Scholar y Redalyc. Además, se revisó la bibliografía de los artículos para localizar nuevas investigaciones, asegurando una continuidad en los estudios y un rigor metodológico.

\section{CLÍNICA}

El SMP no tiene un cuadro clínico característico. Se pueden describir una serie de signos y síntomas que son detectados por el médico, difíciles de diagnosticar, ya que pueden ir desde una simple hemoptisis simulada (expectoración de esputo de sangre del aparato respiratorio) o llegando a la autoinoculación de sangre contaminada con VIH (Balparda, 2007). Es característico que los casos clínicos presenten un curso atípico además de unos resultados poco consistentes con la sintomatología establecida por la madre y el niño (APA, 2002).

Según los estudios realizados por Cujiño et al., (2010) se estima que por 100.000 casos en niños menores de 16 años la incidencia por año se calcula entre 0,5 y 2,0. Así mismo, se ha diagnosticado en niños de cualquier edad, además de casos prenatales donde se da la fabricación de síntomas al feto. No obstante, es más habitual en la primera infancia, entre los 4 y 5 años (APA, 2002).

Los datos aportados en los estudios de Criddle (2010), de la Cerda et al. (2006) y Kahan y Yorker (1991) nos muestran que este tipo de maltrato afecta por igual a ambos sexos.

La descripción clínica del SMP varía según los métodos y tácticas que utiliza el perpetrador en cada caso (Rodríguez et al., 2003). Delgado (1997) afirma, que las más habituales son: problemas digestivos, dolor abdominal, hemorragias, manifestaciones neurológicas, cutáneas y nefrourológicas.

No obstante, son diversas las estrategias utilizadas por los padres que causan enfermedades a sus hijos, con el fin de conseguir sus objetivos, entre los que destaca el beneficio secundario, mal establecido, de la hospitalización de su hijo (APA, 2000; Espinosa et al.; Rodríguez et al., 2007).

Según de la Cerda et al. (2006), las estrategias realizadas por la madre se pueden clasificar de la siguiente manera:

- La falsificación de información al equipo 
médico.

- La simulación de signos utilizando pruebas falsas como boicotear la orina añadiendo azúcar o sangre menstrual para su análisis y estudio, falsificar la toma de fiebre administrando antipiréticos o incluso frotando el termómetro para aparentar la presencia de fiebre en el niño.

- La producción de signos de distintas formas: asfixia mediante bolsas de plástico o con las propias manos en la boca del niño, provocación del vómito, sedantes, venenos e incluso erupciones a través de ciertas substancias de carácter altamente irritable a la piel de un niño.

Según Cridle (2010), los métodos utilizados por los perpetradores se dividen en 4 categorías:

- Infecciones, como la introducción de material fecal en heridas, inyectarle orina al niño o introducir heces en catéteres intravenosos.

- Lesiones, ingesta de cuerpos extraños, osteomielitis, repetidas conjuntivitis, fracturas sin sanar y heridas no curadas.

- Generar de distintas maneras el sangrado.

- Envenenamiento, a través de sustancias como Ipecacuana, sal, insulina, laxantes o algún tipo de ansiolítico.

Brahm (2008), afirma que la clínica no concuerda en la mayoría de los casos y los resultados obtenidos en laboratorio son, por lo general, negativos. El interés se centra ante las múltiples exploraciones e ingresos hospitalarios que se realizan continuamente al niño, presentando una patología grave, poco frecuente y no responden correctamente al tratamiento. Por ello, es importante llevar a cabo una evaluación exhaustiva de la conducta de la madre (de la Cerda et al., 2006; Espinosa et al., 2000; Goñi et al., 2008). Según Bools et al. (1994; citados por Rodríguez et al., 2003), es importante ver si hay conductas antisociales y los antecedentes psiquiátricos en la madre.

\section{PERFIL FAMILIAR Y FIGURA DEL PERPETRADOR}

Hay una serie de características familiares que pueden dar lugar a la aparición de dicho síndrome, y es por ello que para establecer el diagnóstico es necesario conocer el perfil de ambos padres (Brahm, 2008).
En la mayor parte de los casos es la madre figura perpetradora (APA, 2002; Brahm, 2008; Cujiño et al., 2010; Espinosa et al., 2000; Fernández-Jaén et al., 2006; Garrote et al., 2008; Meadow, 1982). En el estudio realizado por Rosenberg (1987) con una muestra de 117 casos de SMP, la figura perpetradora era la madre en un 98\% de los casos. La mayoría de los estudios, señalan que el padre, un familiar o un amigo sólo se presentan en el 2\% de los casos (Cujiño et al., 2010; Rosenberg, 1987; Zélaya \& Garda, 1992).

Según Pedreira y Martín-Álvarez (2001) el padre tiene un papel importante en estos casos ya que su forma de actuar es esencial para que la madre obtenga poder para tomar el control del ámbito familiar: mantienen poca relación con su familia y realizan algunas tareas para estar lejos de ésta (Cujiño et al., 2010). Se le conoce, así como padre periférico. Se observa un divorcio emocional debido a las tensiones familiares realizando chantajes emocionales de unos contra otros. Los cónyuges llegan a separarse o divorciarse. Se interesa muy poco si su familia enferma y no presta la atención que merece. Posee un nivel socioeconómico menor que el de la madre.

La dinámica familiar aparenta ser estable, no disfuncional, que resalta por una madre completamente involucrada en la atención que merece el niño y, por otra un padre lejos de causar un conflicto o daño a su hijo (Brahm, 2008; Cujiño et al., 2010).

En la mayoría de los casos, se detectan una serie de problemas conyugales (APA, 2002; Rodríguez et al., 2003; Zelaya \& Garda, 1992) debido a la infidelidad del padre por lo que la madre adopta una actitud de ignorancia del hecho y con la intención de suprimir la relación extramarital de su marido. Para ello, produce la simulación de ciertas enfermedades en su hijo obteniendo para sí tener a su marido cerca de ella (Zélaya \& Garda, 1992).

Forsyth (1996) establece una serie de factores que dan lugar a la aparición del SMP:

1. La madre tiene una historia de rechazo o abuso en su infancia por uno de sus padres o por ambos y una deficiente relación marital. Estos rechazos se siguen dando en la adultez unida a una autoestima baja.

2. Entre la madre y su hijo existe una relación patológica, debido a una participación excesiva. 
3. La madre recibe recompensas por parte del sistema médico junto con un clima de apoyo y mejorando sus sentimientos de aislamiento cuando se le admite en el hospital por la enfermedad de su hijo.

4. Trastornos psicopatológicos en la figura perpetradora: depresiones, tentaciones suicidas, trastornos somáticos, facticios y de personalidad (grupos A y B).

Pedreira y Martín-Álvarez (2001) afirman la presencia de alteraciones de conducta y de la personalidad en estas madres, tales como, conductas obsesivas, rasgos histéricos y una acusada depresión. Se encuentran muy adaptadas a la sociedad llamando la atención por su perfeccionismo y exigencia en sus comportamientos.

Según Rand y Feldman (1999), estas madres se comportan de manera extraña e inadecuada principalmente cuando hay un factor altamente estresante para ellas. Waller (1983; citado por Rodríguez et al., 2003) refiere que los mecanismos disociativos que se dan en estas madres los utilizan para manipular el problema de su hijo en presencia del médico.

Según la APA (2002) pueden presentar trastornos de la personalidad y trastornos somatomorfos. Además, la madre puede presentar un trastorno facticio, junto con el trastorno facticio por poderes, que no sale a la luz mientras la madre induce daños en el niño. Si la figura perpetradora y la víctima colaboran en estas acciones, se puede diagnosticar a la víctima de trastorno facticio.

En el estudio realizado por Enoch (1990), concluye que el factor común de estas mujeres es la denominada mentira patológica condicionada por mecanismos psicológicos de manera consciente e inconsciente. Esta investigación señala que pueden presentar trastornos de personalidad de tipo histriónico y/o límite. Según la APA (2002), esa mentira patológica (pseudología fantástica) se presenta cuando el perpetrador explica las vivencias diarias y la situación del menor. No obstante, cuando confrontan con la madre, éstas niegan haber mentido. Posteriormente, pueden llegar a tener ideas suicidas cuando se descubre el SMP.

Para Libow y Schreier (1986) se puede clasificar la figura perpetradora en tres categorías de acuerdo con las características que presentan:
- Cuidadores (Help Seeker): esta categoría hace referencia a aquellas personas que reclaman atención para su hijo con el fin de explicar el estado de agotamiento que sufren o la falta de habilidad a la hora de hacerse cargo de su hijo. Son amas de casa con fuertes problemas familiares, personas solteras e incluso que se ocupan de cuidar a enfermos.

- Inductores activos (Active Inducer): son madres con manifestaciones ansioso-depresivas que se caracterizan por la utilización de tácticas altamente dramáticas para inducir enfermedades en el menor. Padecen estados disociativos y fuertes rasgos paranoides. También suelen mostrar una necesidad de control ante las actuaciones médicas debido a su creencia sobre la incompetencia de éstos.

- Doctoadictos (Doctor Addict): se caracteriza por una obsesión basada en creer que sus hijos están enfermos y que, por lo tanto, la administración de un tratamiento resulta esencial.

Meadow (1989) describe a la figura perpetradora dentro de estas categorías:

- Enfermedades percibidas (perceived illnes): la madre explica que su hijo padece ciertos síntomas que sólo ellas ven y que consecuentemente no se encuentran conformes con el tratamiento administrado. En esta primera clasificación no se describe como una forma de abuso excepto cuando la madre se excede en las intervenciones que se hacen al niño poniendo en peligro su vida.

- Visitadores médicos (doctor shopping): la madre va de médico en médico explicando la enfermedad de su hijo. Debido a que muchos de ellos rehúsan a realizar el estudio íntegro que la madre solicita, acude a otros médicos como si fuese la primera vez que asiste a un especialista.

- Invalidez forzosa (enforced invalidism): corresponde a una madre que exagera la discapacidad que presenta el niño y en el caso de no padecerlo ella insiste en explicar que si y que por lo tanto necesita una atención especial.

Monteleone (1994) relaciona las descripciones realizadas por Libow y Schreir en 1986 y la clasificación 
de Meadow de 1989, afirmando que la clasificación de "cuidadores" sería proporcional a la de "enfermedades percibidas" y "doctoradictos" con "visitadores médicos".

Según de la Cerda et al. (2006) podemos diferenciar la conducta de la madre desde dos puntos de vista:

- Madre simuladora: cuyo objetivo se basa en obtener una ayuda para sí o para su hijo.

- Madre con condicionante psicológico: caracterizada por continuos engaños y llamadas de atención al médico sobre su hijo de manera compulsiva, queriendo conseguir la etiqueta de madre ideal (de la Cerda et al., 2006).

Es frecuente que en sus antecedentes personales estas madres hayan sido víctimas de malos tratos (APA, 2002; de la Cerda et al., 2006; Goñi et al., 2008) o han padecido un SM en su infancia (de la Cerda et al., 2006; Goñi et al., 2008) o abusos sexuales (APA, 2002).

Distintos autores reportan información sobre una base común en casos de SMP explicando que éstas anteriormente sufrieron cierta adicción al alcohol y ciertas sustancias (Black, 1981; Feisher \& Ament, 1977; Fialkov, 1984; Griffith, 1988; Hosh, 1987; Palmer \& Yoshimura, 1984; Stankler, 1977; citados por Rodríguez et al., 2003).

Goñi et al. (2008), clasifican las características generales del perpetrador de la siguiente manera:

- $\quad$ La edad media de las madres es de 30 años.

- Nivel socioeconómico bajo.

- Carecen de empleo fuera del hogar o trabajo inestable.

- Antecedentes familiares de maltrato.

- Familia desestructurada.

- Existencia de trastornos psíquicos o de personalidad.

- Actitud colaboradora con el personal médico.

- Actitud despreocupada con relación a la situación del niño y oscilante entre indiferencia y excesiva preocupación.

Balparda (2007) las define como muy comprometidas y que aceptan la administración de un tratamiento por parte de los sujetos de la institución. Sin embargo, su interés se decanta más por las atenciones médicas que por el paciente (Maida et al., 1999).

Goñi et al. (2008) lo explica como una preocupación desproporcionada, y que además se ocupa de manipular e inducir a los profesionales del ámbito de la salud el sometimiento de grandes intervenciones al niño.

Según Maida et al. (1999), el objetivo esencial se centra en tener buenas relaciones con el equipo sanitario (Criddle, 2010; Rodríguez et al., 2003) y consecuentemente el aparente interés le hace no tener sospecha ante la probabilidad de ser agresor. Frecuentemente la madre tiene conocimientos sobre medicina (APA, 2002; Berry 2008; Brahm, 2008; Fernández-Jaén et al., 1998; Goñi et al., 2008; Rodríguez et al., 2003) así como, conocimientos sobre el funcionamiento del hospital (APA, 2002).

Pedreira y Martín-Álvarez (2001) señalan que en sus antecedentes personales cuidaron algún miembro familiar con enfermedad de carácter crónico. Esto les proporciona conocimientos sobre lo que puede ocurrir por un exceso de medicación, además de las consecuencias que se pueden dar y las alternativas de solución que se pueden realizar.

De la misma manera, Rodríguez et al. (2003) explican que estas madres están en el hospital atendiendo a su hijo constantemente, proporcionando un cuidado excesivo en el niño.

En esta misma línea Rand y Feldman (1999) explican que el perpetrador suele sentirse especial catalogándose, como una persona heroica por la atención prestada a su hijo, cuyo fin se basa en llamar la atención de su alrededor, a sus amigos, familia y personas cercanas a ésta.

\section{CRITERIOS DIAGNÓSTICOS}

Fernández-Jaén et al. (1998) explica que en ocasiones el diagnóstico es definitivo cuando se identifican sustancias tóxicas en la sangre o en la orina, cuando se ve como la madre induce directamente la enfermedad al niño e incluso los resultados de laboratorio están alterados.

Según Karlin (1995), cuando se produce una enfermedad crónica en el niño derivado del SMP, los resultados de las pruebas de laboratorio se ven alteradas. De esta manera resulta imposible averiguar el momento real de la aparición de dicha patología, e incluso si la sintomatología es inventada dificultando así 
el diagnóstico definitivo. Esto supone un estudio prolongado en el tiempo en el que la duración entre la consulta y dicho diagnóstico se establece en más de 6 meses (Cujiño et al., 2010; de la Cerda et al., 2006; Rodríguez et al., 2003) pudiéndose prolongar incluso en años (Rodríguez et al, 2003). Sin embargo, Sheridan (2003; citado por Criddle, 2010) informa que el tiempo entre los síntomas y la aparición del diagnóstico se da alrededor de los 21,8 meses.

A través de la denominada prueba de separación se comprueba que los síntomas en el niño remiten cuando la madre está lejos de él (de la Cerda et al., 2006). Sin embargo, Pankratz (2010) concluye que los menores no deben separarse de sus madres a la hora de diagnosticar el caso y que sólo debe realizarse cuando corren un grave peligro y como prueba forense.

Sería esencial vigilar ciertas conductas de la madre a través de cámaras de video, aunque al ser necesario la autorización previa, pero tal y como señala Cujiño et al. (2010) la mayoría de los padres no lo aceptan. En la mayoría de los estudios el diagnóstico se establece en un promedio entre 30 y 40 meses, desde la aparición de los primeros síntomas. La demora en el diagnóstico puede generar graves consecuencias en el menor.

Los criterios que mejor definen al SMP son la voluntad, la intención, la falta de un beneficio consciente (a diferencia de la simulación que está motivado por la búsqueda de un beneficio económico y legal evidente) (APA, 2002; de la Cerda et al., 2006; Garrote et al., 2008) y la falta de control sobre su propia conducta ya que actúan de manera compulsiva y las manipulaciones las realizan de manera consciente pero no sus motivaciones.

(Tabla 1. Semejanzas y diferencias entre la Simulación, el Síndrome de Munchausen/ Síndrome de Munchausen por Poderes.

\begin{tabular}{lcc}
\hline & Producción síntomas & Motivación \\
\hline Simulación & Consciente & Consciente \\
\hline SM / SMP & Consciente & Inconsciente \\
\hline
\end{tabular}

Tomada de Jiménez-Perianes, 2011.

Para realizar el diagnóstico habría que tener en cuenta las características anteriormente citadas y según Cujiño et al. (2010), Fernández- Jaén et al. (1998), Garrote et al. (2008) y Zélaya y Garda (1992) las siguientes señales de alarma:

- Enfermedades sin aparente explicación, cuya duración es prolongada en el tiempo y cuya naturaleza es tan extraña que el equipo médico llega a afirmar no haber visto nada similar antes.

- Signos y síntomas poco habituales y cuyo sentido clínico es extraño. Además, esta sintomatología, se presenta en algunas ocasiones y coincide con la presencia de la madre (Criddle, 2010).

- Los resultados de varios diagnósticos realizados son contradictorios ya que se producen discrepancias entre el historial del niño, los resultados clínicos y el bienestar del menor.

- Aplicación de tratamientos que resultan ineficaces o que demuestra gran intolerancia.

- Aparición de convulsiones que no responden al tratamiento pautado por el médico.

- Presencia de alergias a ciertos alimentos o fármacos.

- Madres menos preocupadas que los médicos por la enfermedad de su hijo y, cuya estancia en el hospital es constante, aunque sea en breves periodos de tiempo. Además, muestran gran colaboración en el cuidado del niño y por lo general mantienen estrechas relaciones con el personal de sanidad. Son madres que tienen un historial de enfermedades similares a las de su hijo/a.

- El padre no está presente.

- Dentro de la familia se han dado enfermedades de hermanos inexplicables e incluso muertes súbitas, además de la presencia de patologías graves y diversas en muchos de sus miembros, por lo que la madre puede tener experiencia previa en lo relacionado con la medicina o enfermería.

De acuerdo con el DSM-IV-TR (APA, 2002) el Trastorno Facticio por Poderes establece los siguientes criterios con propuesta de investigación: (a) producción o simulación intencionada de signos o síntomas físicos o psicológicos en otra persona que se encuentra bajo el cuidado del perpetrador, (b) la motivación que define al perpetrador es el deseo de asumir el papel de paciente a través de otra persona,(c) no existen incentivos externos que justifiquen este comportamiento (por ejemplo, una compensación económica) y, (d) el comportamiento no se explica mejor por la presencia de otro trastorno mental. 
Tabla 2. Cuadro de decisión para el diagnóstico de Síndrome de Munchausen en la infancia y la adolescencia.

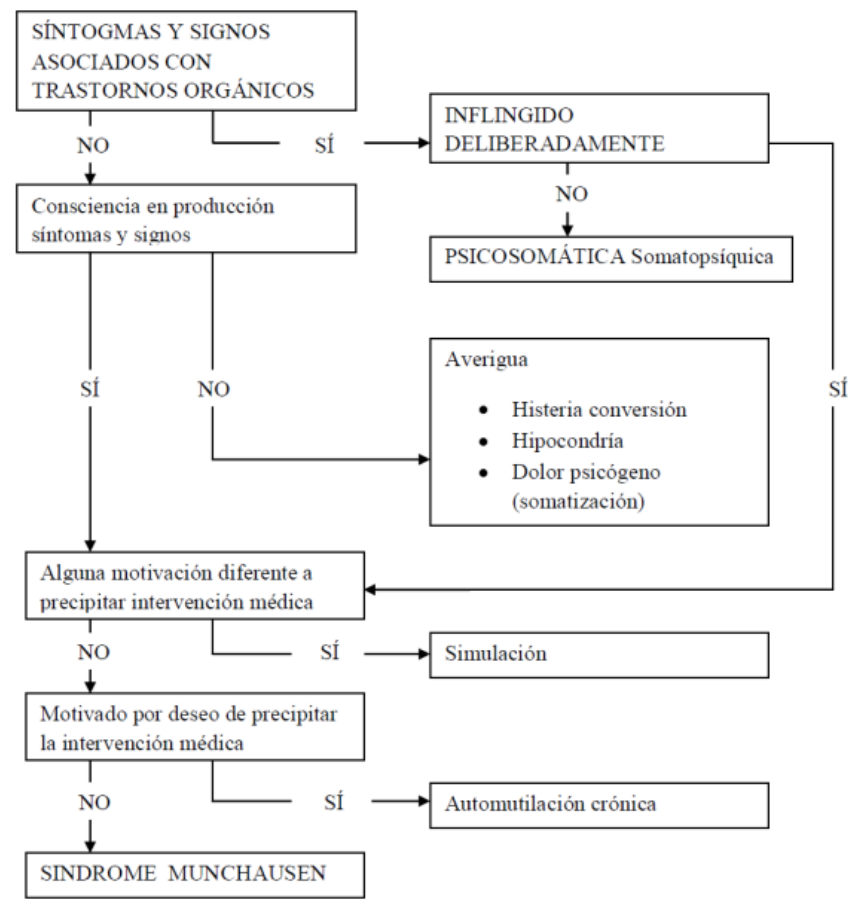

Tomada de Pedreira y Martín-Álvarez, 2001.

En el DSM-5 (APA, 2013) se denomina Trastorno Facticio aplicado a otro cuyos criterios son: (a) falsificación de signos o síntomas físicos o psicológicos, o inducción de lesión o enfermedad, en otro, asociada a un engaño identificado, (b) se presenta a otro individuo (víctima) frente a los demás como enfermo, incapacitado o lesionado, (c) el comportamiento engañoso es evidente incluso en ausencia de recompensa externa obvia y, (d) el comportamiento no se explica mejor por otro trastorno mental como el trastorno delirante u otro trastorno psicótico. Hay que especificar si ha sido un episodio único o se presenta de manera recurrente.

\section{DIAGNÓSTICO DIFERENCIAL}

Es importante establecer una distinción entre el SM dado en adultos, que se ocupan de fabricar sus enfermedades y producirse ellos mismos los síntomas y signos, y el SMP que se presenta en los adultos que inducen enfermedad al menor que se ocupan de cuidar, generando un tipo de maltrato en el niño (Rodríguez et al., 2003).

Según la APA (2002), el trastorno facticio por poderes se diferencia de una enfermedad médica o un trastorno mental en aquel individuo va buscando ayuda terapéutica. El trastorno se puede diferenciar de los abusos físicos o sexuales que no se relacionan con la intención de asumir de forma indirecta el papel de paciente.

El trastorno facticio por poderes y la simulación se diferencian en que en ésta la producción de la sintomatología está motivada por una ganancia externa mientras que en el trastorno facticio por poderes no es así. Los sujetos que se ocupan de simular enfermedades solicitan ingreso en un hospital para que alguien les cuide, pero la producción de la sintomatología se da para obtener una compensación.

Brahm (2008) explica que el diagnóstico diferencial es amplio y abarca desde una alergia habitual o una posible dermatitis, hasta la muerte súbita, pasando por enfermedades tales como: epilepsia, osteomielitis, epilepsia o hiperinsulismo, entre otros.

Porter et al. (1994), señalan que la aparición recurrente de partos prematuros debe ser añadido al diagnóstico diferencial.

\section{CONSECUENCIAS Y REPERCUSIONES}

Rodríguez et al. (2003) clasifican en diversos niveles las consecuencias del SMP: físicas, emocionales, psicológicas y sociales. Las víctimas de este maltrato poseen un pronóstico desfavorable: pueden desarrollar problemas emocionales y comportamentales que pueden generar dificultades atencionales y de concentración, repercutiendo negativamente en el ámbito escolar (APA, 2002; Fernández-Jaén et al., 1998).

En diferentes estudios retrospectivos, los sujetos que presentaron SMP en la infancia sufrieron secuelas emocionales y físicas por lo que en su adultez se sentían inseguros, evitando someterse a tratamiento médico y además presentaban síntomas característicos de estrés post-traumático (APA, 2002; Brahm, 2008; Libow, 1995). Así mismo, expresaban un fuerte rencor hacia las madres causantes de tal abuso, sin embargo, hacia sus padres sentían compasión, quienes adoptaron una actitud pasiva o fracasaron en la protección de sus hijos.

Pedreira y Martín-Álvarez (2001) añaden que estos niños sufren problemas en su desarrollo psicosocial con cierta cronicidad. No tienen carencias a nivel material sino en su ámbito afectivo y emocional como problemas a la hora de expresar su afectividad o un exceso de emoción expresada. También se pueden dar dificultades en las relaciones y vínculos con las figuras 
de apego, así como grandes cambios en su rol social. Si se une una cierta alexitimia junto con el desarrollo psicosocial descrito anteriormente y etiquetado como encerramiento relacional con aislamiento social producen que a lo largo del funcionamiento psicosocial puedan encontrarse ciertos problemas que necesiten una vía de expresión.

McGuire y Feldman (1989) han estudiado casos en los que las víctimas del SMP participan en el engaño del perpetrador, y de acuerdo con Murray (1997) y Fernández Jaén et al. (1998), también pueden desarrollar un SM en la edad adulta.

Para Karlin (1995), el niño puede llegar a ver el amor de su madre como un vínculo dependiente del inicio de la enfermedad por lo que el menor entra a formar parte del engaño o provocándose él mismo determinados síntomas, para protegerse a sí mismo o por miedo a ser abandonado. Cuando el niño se identifica con la enfermedad y la usa como vía de expresión y medio comunicativo con la madre, llega a convertirse en paciente del SM: de víctima a perpetrador de sí mismo. E incluso, se puede convertir, en un futuro, también en perpetrador del SMP, realizando esto con sus hijos. La conducta del menor frente a la conducta de la madre se define así: "el niño comienza con un rechazo, con negativismo y ansiedad, y luego progresivamente entra en la pasividad" (Karlin, 1995, p. 195).

Tal como señala Lynch en 2006, tener una historia previa de fingir o inducir a la enfermedad propia de uno, como se indica con el SM, es un factor de riesgo en las personas que luego desarrollan SMP.

Karlin (1995) explica que los niños que tienen menos de 6 años pueden creer que la responsabilidad de sus enfermedades es de ellos mismos y de este modo altera el concepto de sí mismo que tiene el niño y su fuerza yoica. Este mismo autor, comenta que las relaciones con sus iguales en niños mayores son pobres debido a la falta escolar por la cantidad de hospitalizaciones.

A largo plazo las consecuencias afectan tanto al niño que sufre los daños como para el resto de los menores que viven en la misma casa (Cujiño et al., 2010), encontrando muertes de hermanos cuyas causas son inciertas, denominadas como muerte súbita (de la Cerda et al., 2006).

Es complicado averiguar el total de muertes que se dan en las víctimas del SMP, sin embargo, varios estudios confirman que está en torno al 6\% (Criddle, 2010), al 9\% (Cujiño et al, 2010) o 10\% (Criddle, 2010; Precey, 1998; citado por Berry, 2008). Las causas de muerte que se dan con más frecuencia son asfixias y por la administración de veneno, cuya tasa de mortalidad está en torno al 33\% (Criddle, 2010).

Se ha puesto de manifiesto que cuando muere el primer niño víctima de este abuso puede darse el síndrome en los demás menores de la casa. En aquellos casos en los que los niños que no sufrieron daños físicos volvieron con sus padres, se presentaron situaciones de maltrato en el 17\% de los casos. Sheridan (2003) realizó un estudio en el que el 7,3\% de los niños sufrieron lesiones durante un largo periodo de tiempo o secuelas de carácter permanente y un $6 \%$ de ellos murieron.

\section{INTERVENCIÓN}

Para Loredo-Abdalá y Bobadilla (1987) y LoredoAbdalá et al. (1990), el SMP debe tener un tratamiento integral: intervención médica, en ocasiones quirúrgica, del menor, tratamiento por parte del psiquiatra a toda la familia, evaluación social, y, siempre teniendo en cuenta el aspecto jurídico que puede conllevar cada caso particular.

Fernández-Jaén et al. (1998) destacan que la actitud que se lleva a cabo en estos casos se centra primeramente en la utilización del equipo multi e interdisciplinario en cuyo caso participan trabajadores sociales, psicólogos y el psiquiatra infantil.

Según Garrote et al. (2008), este equipo se ocupa de valorar el riesgo que corre el paciente y se procedería con el diagnóstico en el momento que el niño esté protegido. Rodríguez et al. (2003) señalan que es importante una intervención terapéutica para proporcionar ayuda psicológica al niño y a su madre, así como, una protección jurídica del caso resulta esencial para el niño y otros menores en los que su vida corre un gran riesgo. Por todo ello, en consonancia con De la Cerda et al. (2006), se debe llevar a cabo una serie de actuaciones al respecto desde distintas áreas:

- Actuación del equipo de Salud Mental.

- Actuación de los trabajadores sociales.

- Adoptar medidas legales.

- Confrontación con el ámbito familiar. 


\section{CONCLUSIÓN}

En la mayoría de los estudios coinciden en señalar que la madre es la autora de los hechos, sin embargo, el padre aparece en una proporción mucho menor. Los estudios de casos que se han realizado con respecto a esta figura señalan que el porcentaje de la madre como figura perpetradora se da en torno al 98\%, mientras que el padre e incluso persona que se ocupa de cuidar del menor aparece en un 2\%. La presentación de ambos progenitores como perpetradores está en un 6,7\% de los casos que se han investigado.

En la mayor parte de los casos, la madre posee antecedentes psiquiátricos. Generalmente, presentan rasgos ansiosos-depresivos, así como conductas obsesivas y/o rasgos histéricos. La mayoría de los estudios, demuestran que en muchas de estas madres existe la posibilidad de presentar alteraciones de conducta y de la personalidad, insistiendo también en una acusada depresión. Señalan que incluso pueden presentar una patología disociativa, explicándose por la simultaneidad de pensamientos contradictorios debido en gran parte a que en el acto de maltratar a su propio hijo se sienten madres excelentes.

La presencia de los problemas conyugales es otro de los criterios presentes. Algunos autores explican que el padre al tener relaciones extramaritales, la madre utiliza este tipo de maltrato a su hijo para conseguir llamar la atención del padre y ser el vínculo perfecto para que esté más tiempo con ella y apartarle de la relación que tiene fuera de su matrimonio. Hay que señalar, la actitud del padre pasiva, mostrando indiferencia ante la situación que se presenta en la familia y por lo tanto, se desliga del problema, además de tener una deficiente relación conyugal con su mujer, apenas están en casa y no se preocupan de su hijo.

El SMP no tiene un cuadro clínico característico. Se pueden describir una serie de signos y síntomas que son detectados por el médico, difíciles de diagnosticar, presentando un curso atípico además de unos resultados poco consistentes con la sintomatología presentada.

El diagnóstico es definitivo cuando se identifican sustancias tóxicas en la sangre o en la orina, cuando se ve como la madre induce directamente la enfermedad al niño e incluso los resultados de laboratorio están alterados mostrando datos atípicos.

Así mismo, es importante conocer el perfil psicopatológico y de personalidad que puede mostrar la figura perpetradora con el objetivo de establecer un diagnóstico correcto.

Es crucial establecer el diagnóstico correcto de manera temprana con el objetivo de proteger al menor, evitando secuelas importantes e impedir su fallecimiento.

Una vez identificado el SMP, se debe de llevar a cabo un tratamiento integral a nivel clínico y legal.

Se propone la creación de un protocolo específico de actuación que detalle de manera clara los criterios a seguir para abordar un posible caso, con el objetivo de establecer un correcto trabajo inter/multidisciplinar.

\section{REFERENCIAS}

American Psychiatric Association. (2002). Diagnostic and Statistical Manual of Mental Disorders. DSM-IV-TR. (4th ed., textrev). Washington DC: Author.

American Psychiatric Association. (2013). Diagnostic and statistical manual of mental disorders. DSM-5. (5th edn.). https://doi.org/10.1176/appi.books.9780890425596

Asher, R. (1951). Münchhausen syndrome. The Lancet, 6650 (257), 339-41. https:/ / doi.org/10.1016/S01406736(51)92313-6

Balparda, J.K.( 2007).El barón enfermo: síndrome de Munchausen. Medicina UPB, 26, 23-32. Obtenido de URL:

http:/ / redalyc.uaemex.mx/redalyc/pdf/1590/15901498 9003.pdf.

Berry, L. (2008). Understanding Munchausen Syndrome by Proxy. Academic Journal, 14 (4), 4-13.

Bools, C., Neale, B., \& Meadow, R. (1994). Munchausen syndrome by proxy: a study of psychopathology. Child Abuse \& Neglect, 18, 773-788. doi: 10.1016/01452134/94.00041-1

Brahm, P. (2008). Maltrato infantil oculto: Síndrome de Munchausen por Poderes. Obtenido de URL:http://www.medicinafamiliaruc.cl/html/articulos /120.html

Criddle, L. (2010). Monsters in the closet: Munchausen syndrome by proxy. Critical Care Nurse, 30, 46-55. doi: $10.4037 / \operatorname{ccn} 2010737$

Cujiño, M.F., Dávila, A., Sarmiento, M.M., Villarreal, M.I. \&Chaskel R. (2010). Síndrome de Munchausen por poder. Pediatría, 43, 41-50.

De la Cerda F., Goñi, T. \& Gómez de Terrenos, I. (2006). Síndrome de Munchausen por poderes. Cuadernos de medicina forense, 12, 47-55. doi.:/10.4321/S113576062006000100004 
Delgado, A. (1997). Sindrome de Münchausen por Poderes. Niños maltratados. Madrid: Díaz de Santos.

Enoch, D. (1990). Hysteria, malingering, pseudological fantastica, cancer syndrome, prison psychosis and Munchausen's syndrome. Principles and Practice of Forensic Psychiatry, London: Churchill Livingstone.

Espinosa, A., Figueras, B., Mendilahaxon, J. \& Espinosa, A. (2000). Síndrome de Münchausen: Un reto para el clínico. Revista Cubana de Medicina, 39, 228-37. Obtenido de URL: http:/ / scielo.sld.cu/scielo.php?pid=S003475232000000400005\&script=sci_arttext.

Fernández-Jaén, A., Martínez- Bermejo, A., López-Martín, V. \& Pascual- Castroviejo, I. (1998). Síndrome de Munchausen por poderes: presentación de un caso con epilepsia. Revista de neurología, 26, 772-774. Obtenido de URL:

http://www.neurologia.com/pdf/Web/26153/e153077 2.pdf

Forsyth, B. W. C. (1996). Munchausen syndrome by proxy. Child Adolesc Psychiatry, 1048-1054.

Garrote, N., Indart, J., Puentes, A., Smith, M., del Bagge, P. \& Pérez, M. (2008).Síndrome de Munchausen por poder y manifestaciones de supuesto evento de aparente amenaza a la vida. Archivos argentinos de pediatría, 106, 4753. Obtenido de URL:

http://www.scielo.org.ar/scielo.php?pid=S0325007520 08000100011\&script=sci_arttext

Gómez de Terreros, I., Gómez de Terrenos, M., Serrano, M., Jiménez, AV., Sánchez, M., Salazar, N., López, A.M. \& Malo, J.M. (1996). Ingestión Reiterada de Cuerpos Extraños. Forma Inusual de Presentación del Síndrome de Munchausen por poderes. Child Abuse \& Neglect, 20, 613-620. doi: 10.1016/0145-2134/96/00048-8

Goñi,T., Martínez, Mà.J., de la Cerda, F. \& Gómez de Terrenos, I. (2008). Síndrome de Munchausen por poderes. Servicio de Pediatría General., 68, 609-11. doi: $10.1157 / 13123295$

Jiménez-Perianes, A. (2011). Apuntes de Psicología de la Salud. Comunicación personal.

Kahan, B., \& Yorker, B. C. (1991). Munchausen syndrome by proxy: Clinical review and legal issues. Behavioral Sciences \&o the Law, 9, 73-83.

Karlin, N. J. (1995). Munchausen syndrome by proxy. Brattleboro Retreat Psychiatry Review, 4. Obtenido de URL: www.bratretreat.org/brpr./v4n1.html

Le Heuzey, M. (2010). Munchausenby proxy: child's psychiatrist point of view. Archives de Pédiatrie, 17, 642643.

Libow, J. (1995). Munchausenby proxy victims in adulthood: A first look.Child Abuse \& Neglect, 9, 1131 1142. doi: 0145-2134/95/00073-9
Libow, J. A., \& Schreier, H. A. (1986). Three forms of factitious illness in children: when is it Munchausen syndrome by proxy?. American Journal of Orthopsychiatry, 56, 602-611.

Loredo-Abdalá, A., \& Bobadilla, M. M. (1987). Síndrome del niño maltratado: aspectos físicos, emocionales y sociales. Rev Mex Pediatr, 54, 123-131.

Loredo Abdalá, A., Oldak Skvirsky, D., Carbajal Rodrigues, L., \& Reynes Manzur, J. (1990). Algoritmo del niño maltratado a su ingreso a un centro hospitalario pediátrico de tercer nivel. Bol. méd. Hosp. Infant. Méx, 47, 91-5.

Maida, S., Molina, P. \& Carrasco, C.H. (1999). Síndrome de Munchausen-por-poder: un diagnóstico a considerar. Revista Chilena de Pediatría., 70, 215-220. doi: 10.4067/S0370-41061999000300007

Martínez, A. \& De Paúl, J. (1993). Tipología del maltrato infantil. En A. Martínez \& J. De Paúl (Eds.), Maltrato y abandono en la infancia (pp.25-30). Barcelona: Martínez Roca.

McGuire, T. L., \& Feldman, K. W. (1989). Psychologic morbidity of children subjected to Munchausen syndrome by proxy. Pediatrics, 83(2), 289-292.

Meadow, R. (1977). Munchausen syndrome by proxy the hinterland of child abuse. The Lancet, 310(8033), 343345. https://doi.org/10.1016/S0140-6736(77)91497-0

Meadow, R. (1989). ABC of child abuse. Munchausen syndrome by proxy. BMJ: British Medical Journal, 6693, 248-299.

Murray, J. B. (1997). Munchausen syndrome/Munchausen syndrome by proxy. The Journal of psychology, 131, 343352.

Pankratz, L. (2010). Persistent problems with the "separation test" in Munchausensyndromeby proxy. Journal of Psychiatry \& Law, 38, 307-323.

Pedreira, J.L., Palanca, I., Sardinero, E. \& Martín, L. (2001). Los trastornos psicomáticos en la infancia y adolescencia. Revista de psiquiatría y psicología del niño y del adolescente, 3, 26-51.

Pedreira, J.L. \& Martín- Álvarez (2001). Síndrome de Munchausen en la infancia y adolescencia. En J.L. Pedreira \& J. Tomas (Eds.), Condicionantes psicosomáticos y su tratamiento en la infancia y la adolescencia: agresividad, violencia, insomnio, medios de comunicación, sida (pp. 193-208). Barcelona: Laertes.

Porter E., Heitsch, M. \& Miller, D. (1994). Munchausen syndrome by proxy: unusual manifestations and disturbing sequelae. Child Abuse \& Neglect, 18, 789-94. doi: 10.1016/0145-2134/00045

Rand, D. C., \& Feldman, M. D. (1999). Misdiagnosis of Munchausen syndrome by proxy: A literature review 
Cedenilla, M. \& Jiménez-Perianes, A. (2020). Aproximación teórica al Síndrome de Munchausen por Poderes. Behavior \& Law Journal, 6(1), 1-11.

and four new cases. Harvard Review of Psychiatry, 7(2), 94101.

Rodriguez, L., Gómez, M., \& Del Pilar, A. (2003). Estado del arte sobre el Síndrome de Munchausen por Poderes. Unviersitas Psychologica, 2, 187-198.

Rosenberg, D. A. (1987). Web of deceit: a literature review of Munchausen syndrome by proxy. Child abuse \& Neglect, 11, 547-563.

Shapiro, M. \&Nguyen, M. (2011). Psychological sequelae of Munchausen's syndrome by proxy. Child Abuse \& Neglec, 35, 87-88. doi: 10.1016/j.chiabu.2010.10.005

Shaw, R. J., Dayal, S., Hartman, J. \&De Maso, D. R. (2008). Factitious Disorder by Proxy: Pediatric Condition Falsification. Harvard Review of Psychiatry, 16(4), 215224. doi: $10.1080 / 10673220802277870$
Sheridan, M. S. (2003). The deceit continues: an updated literature review of Munchausen syndrome by proxy. Child abuse \& neglect, 27, 431-451.

Sugandhan, S., Gupta, S., Khandpur, S., Khanna, N., Mehta, M. \&Inna, P. (2010). 'Munchausen syndrome by proxy' presenting as battered child syndrome: a report of two cases. International Journal of Dermatology, 49, 679683.

Verity, C.J., Winckworth, C., Burman, D., Stevens, D. \& White, R.J. (1979). Polle syndrome: children of Munchausen. British Medical Journal, 2, 422-423.

Zélaya,E. \&Garda, O. (1992). Síndrome de Munchausen por "Poderes". Reporte de un caso en nuestro País y una advertencia al Médico Pediatra. Honduras Pediatría, XV,14-16. 